\title{
Appropriateness of the Use of Navigation System in Total Knee Arthroplasty
}

\author{
Suk Han Jung, MD, Myung Rae Cho, MD, Suk-Kyoon Song, MD \\ Department of Orthopedic Surgery, Daegu Catholic University Medical Center, Daegu, Korea
}

Background: The aim of this study was to evaluate the difference between the planned and verified actual values in total knee arthroplasty (TKA) performed using a navigation system.

Methods: Sixty patients who underwent primary TKA for knee pain from March 2018 to July 2018 were included in this study. All patients underwent TKA using the latest version of a computer navigation system (Kick ver. 2.6). All TKA procedures were performed by the same surgeon. The appropriateness of the use of navigation system in each of the several steps during the operation was investigated. Implant size was assessed using a preoperative template and after registration of landmarks with the navigation system. Intraoperative measurement was conducted using a femoral sizing implant apparatus. The difference between the planned value based on the navigation system and the actual cutting value was investigated. Intraoperatively confirmed hip-knee-ankle angle was also compared to the angle measured at postoperative 3 months.

Results: The average time spent on the registration process was 242 seconds (range, 205-345 seconds). Intraoperative femoral component size tended to be smaller than the size recommended by the navigation system. A significant difference between the planned distal femoral cutting level $(9.08 \pm 0.40 \mathrm{~mm})$ and the verified actual cutting level $(9.87 \pm 1.39 \mathrm{~mm})$ was identified $(p<0.05)$. The difference between the planned lateral and medial tibial resection levels $(10.12 \pm 0.34 \mathrm{~mm}$ and $4.47 \pm 2.17 \mathrm{~mm}$, respectively) and the verified actual lateral and medial tibial resection levels $(9.07 \pm 1.45 \mathrm{~mm}$ and $3.48 \pm 2.00 \mathrm{~mm}$, respectively) was statistically significant. Distal femoral cutting angle in sagittal plane was significantly different but femoral and tibial cutting angles showed no significant difference between the planned and verified values. At full extension, the average coronal alignment of the implant recorded after insertion of the actual implant using the navigation system was $0.23^{\circ} \pm 0.51^{\circ}$ varus and showed no significant difference from the alignment measured at postoperative 3 months $\left(0.45^{\circ} \pm 0.58^{\circ}\right)$.

Conclusions: When performing navigation-assisted TKA, surgeons should aware that frequent errors can occur on the femoral cutting level, tibial cutting level, and implant sizing despite its reported advantage in defining the mechanical limb axis.

Keywords: Total knee arthroplasty, Navigation, Osteoarthritis, Knee

To improve the durability of total knee arthroplasty (TKA), several modifications have been made to the implant design and surgical instrumentation. ${ }^{1,2)}$ The mechanical hipknee-ankle (HKA) axis, implant size, and soft-tissue ten-

Received November 11, 2019; Accepted January 20, 2020

Correspondence to: Myung Rae Cho, MD

Department of Orthopedic Surgery, Daegu Catholic University Medical

Center, 33 Duryugongwon-ro 17-gil, Nam-gu, Daegu 42472, Korea

Tel: +82-53-650-4272, Fax: +82-53-626-4272

E-mail:cmr0426@cu.ac.kr sion are crucial factors determining the durability of TKA. The alignment of the knee joint is assessed in the frontal, sagittal, and horizontal planes and defined by anatomically relevant landmarks and axes. Accurate evaluation of these axes and planes is important to achieve an excellent limb alignment, thereby increasing the implant longevity. To ensure accurate alignment of prostheses in the sagittal and frontal planes, the extramedullary femoral rod was promptly replaced by the intramedullary rod, ${ }^{3)}$ which is still used as a reference. Even experienced surgeons need to use this instrument to locate the prostheses placed be- 
Jung et al. Appropriateness of the Use of Navigation System in Total Knee Arthroplasty

Clinics in Orthopedic Surgery • Vol. 12, No. 3, $2020 \bullet$ www.ecios.org

yond the acceptable range with an excessive coronal malalignment (varus and valgus) or excessive posterior or anterior tibial slope. ${ }^{4)}$ However, traditional instrumentation might be insufficient to achieve preoperative objectives. Computerized navigation system for knee replacement surgery was first introduced as early as $1993 .{ }^{5)}$ Navigation facilitates delicate orientation of implants and convenience for precise alignment during surgical procedures. Despite extensive ongoing debates on the accuracy of the intraoperative alignment of the extremity and surgical outcomes with and without a navigation system in primary TKA, some studies have emphasized the superiority of navigation-assisted surgery compared with the conventional methods. Shi et al. ${ }^{6}$ analyzed 15 different trials among the 111 navigated TKA procedures and found substantially better outcomes with navigated TKA compared with conventional TKA. Similarly, Moskal et al. ${ }^{7)}$ analyzed 47 trials among the navigated TKA procedures involving more than 7,000 knees and reported that navigated TKA resulted in better component alignment and clinical outcomes based on the Knee Society and Western Ontario and McMaster Universities Osteoarthritis Index scores. Despite the superiority of navigated surgery over conventional TKA as reported in several studies, its utility has been disputed. Surgeons preferring conventional methods have reported surgical delays when using computer navigation and poor clinical outcomes due to unexpected variations after the planned resection. ${ }^{8-14)}$ We investigated the procedural step associated with differences before and after the planned resection and the step requiring special attention during the use of the navigation system in TKA. The aim of this study was to investigate the difference between the planned values and the verified actual values when using surgical navigation system in TKA.

\section{METHODS}

\section{Patient Selection}

The study included 60 patients undergoing primary TKA for knee pain between March 2018 and July 2018. All the primary TKAs were performed by a single surgeon (MRC) using the latest version of the computer navigation system (Kick ver. 2.6; Brainlab AG, Munich, Germany). The average patient age was 68.9 years (range, $58-81$ years), and the male-to-female ratio was 6:54. All patients manifested Kellgren-Lawrence grade 4 arthritic changes on plain radiographs and Outerbridge 4 cartilage changes intraoperatively. Cemented posterior cruciate ligament-substituting implants (Nexgen; Zimmer Inc., Warsaw, IN, USA) were used in all cases. The standard procedure was conducted using a tourniquet, a standard medial parapatellar approach, and the femur first technique. The computer navigation platform (Kick ver. 2.6) was connected with the pinned navigation marker. Subsequently, all surgical procedures were conducted according to the Brainlab's manual of instructions. Under the guidance of navigation, trial components were inserted and the mediolateral gaps were measured at full extension and at $90^{\circ}$ flexion. Actual prostheses were inserted after a soft-tissue release was performed to minimize the gap difference $(\leq 2 \mathrm{~mm})$. The posterior referencing technique was applied when determining the implant size, and a smaller implant was selected for in-between sizes. All the actual prostheses were inserted when the mechanical HKA angle after the trial component insertion was within $0^{\circ} \pm 2^{\circ}$ based on computer navigation. When an HKA angle was outside the range of $0^{\circ} \pm$ $2^{\circ}$, the value was remeasured subsequent to the soft-tissue release (Fig. 1).

\section{Data Collection and Analysis}

Preoperative assessments included radiographic measurements (anteroposterior [AP] standing knee view, lateral view, Rosen's view, skyline patella-femoral joint view, whole lower extremity scanogram, and AP lateral view scaled to preoperative template measurement) to evaluate preexisting deformity. Patient's sex, age, body mass index (BMI), preoperative function (Oxford score), and anatomic deformity were analyzed by an external investigator (SHJ) who did not participate in surgery. TKA was performed by the same single surgeon (MRC) in all cases. The appropriateness of the navigation system in serial procedures during the operation was investigated step by step. First, we recorded the duration of navigation landmark registration. The planned anterior and distal femoral cutting levels were then recorded and compared with the actual verified values after cutting. The cutting level and alignment of the tibia were recorded and compared with the verified actual values after surgery. The implant size was assessed preoperatively using the template, after landmark registration using the navigation system, and intraoperatively using the femoral sizing apparatus. After implantation, we analyzed and compared the HKA angle, the component alignment, and mediolateral gap in full extension. The HKA angle was determined at 3 months postoperatively in the outpatient department and compared with the intraoperative HKA angle. An outlier was defined as a case beyond the standard $0^{\circ} \pm 2^{\circ}$ and measured twice by an investigator (SHJ) who did not participate in surgery. Another single clinician (SKS) who did not participate in surgery conducted the radiological assessments. The Ox- 
Jung et al. Appropriateness of the Use of Navigation System in Total Knee Arthroplasty

Clinics in Orthopedic Surgery • Vol. 12, No. 3, $2020 \bullet$ www.ecios.org

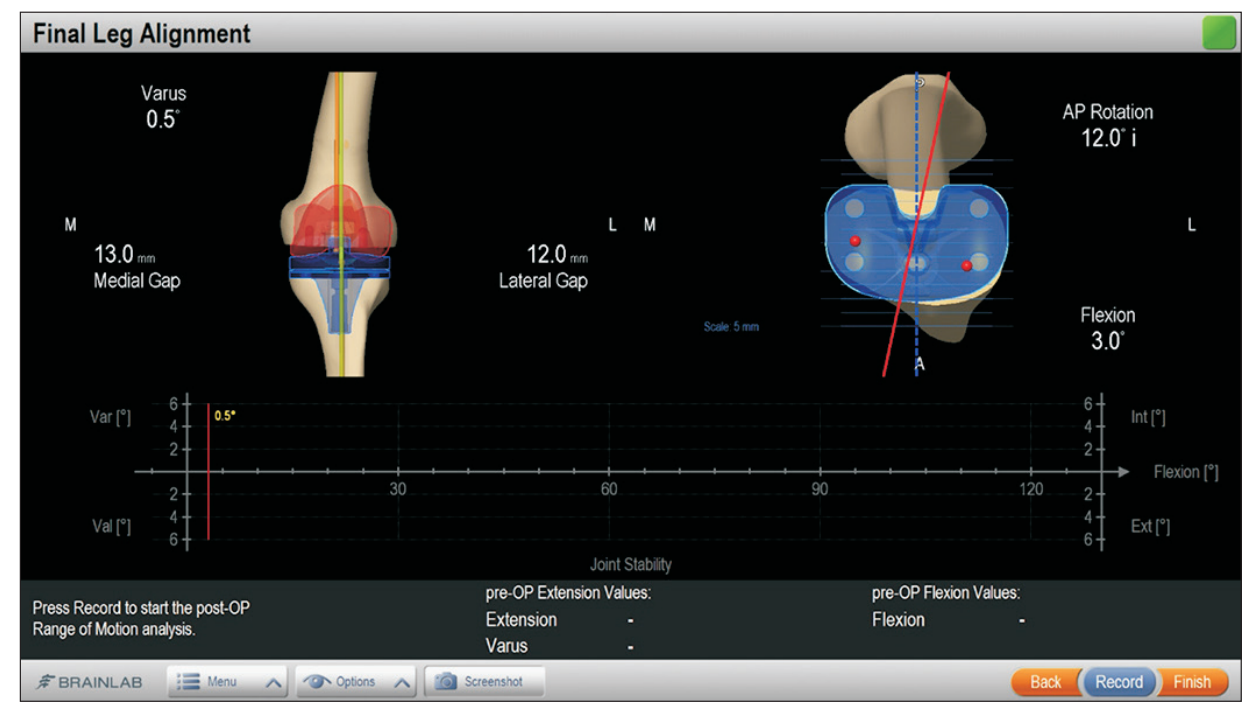

Fig. 1. Intraoperative calculation by the navigation system. ford score was evaluated at postoperative 1 year. Statistical analyses were conducted using IBM SPSS ver. 25.0 (IBM Corp., Armonk, NY, USA) and the statistical significance was set at $p<0.05$.

We conducted this study in compliance with the principles of the Declaration of Helsinki. Institutional Review Board of Daegu Catholic University Medical Center reviewed and approved the protocol of this study (IRB No. CR-19-151), and waived informed consent.

\section{RESULTS}

In the preoperative evaluation, 4 patients had valgus knees and 56 patients had neutral or varus knees. Patients exhibited an average of $7.9^{\circ} \pm 8.6^{\circ}$ of varus (range, $24^{\circ}$ varus to $21^{\circ}$ valgus) alignment in the coronal plane. The average Oxford score determined at 1 year postoperatively was $38.92 \pm 2.76$. The average time spent on the registration process was 242 seconds (range, 205-345 seconds). Femoral component sizes calculated by the navigation system and measured intraoperatively using the femoral sizing apparatus tended to be statistically significantly larger than the preoperative femoral component sizes measured by the template $(p<0.05)$. The intraoperatively selected femoral size was also statistically significantly smaller than the size recommended by the navigation system $(p<0.05)$.

The average planned anterior femoral cutting coronal alignment and the AP shift were neutral (displayed as $0^{\circ}$ angle or $0 \mathrm{~mm}$ cutting width on the screen of the navigation system). The average planned sagittal alignment was $1.2^{\circ} \pm 0.84^{\circ}$ extension (range, $1^{\circ}$ flexion to $2.5^{\circ}$ extension). The actual verified anterior femoral cutting coronal alignment, AP shift, and sagittal alignment after cutting were $0.09^{\circ} \pm 1.65^{\circ}$ external rotation (range, $2.5^{\circ}$ internal rotation to $3.5^{\circ}$ external rotation), $0.17 \pm 0.71 \mathrm{~mm}$ anterior shift (range, $2 \mathrm{~mm}$ anterior shift to $2 \mathrm{~mm}$ posterior shift), and $0.18^{\circ} \pm 0.91^{\circ}$ extension (range, $1.5^{\circ}$ flexion to $1.5^{\circ}$ extension), respectively. Regarding anterior femoral cutting, the difference in sagittal alignment between the planned value and the verified actual value was statistically significant $(p<0.05)$, but there was no significant difference in the coronal alignment and AP shift.

The average planned distal femoral cutting coronal alignment was neutral. The average planned cutting level was $9.08 \pm 0.40 \mathrm{~mm}$ (range, $9-11.5 \mathrm{~mm}$ ). The verified actual anterior femoral cutting coronal alignment and cutting level were $0.13^{\circ} \pm 0.50^{\circ}$ varus (range, $1^{\circ}$ varus to $1^{\circ}$ valgus) and $9.87 \pm 1.39 \mathrm{~mm}$ (range, $8-11.5 \mathrm{~mm}$ ), respectively. Regarding distal femoral cutting, the difference in resection level between the planned value and the verified actual value was statistically significant $(p<0.05)$ but the difference in coronal alignment was not statistically significant (Table 1).

The average planned tibial cutting coronal alignment, sagittal alignment (posterior slope), lateral cutting level, and medial cutting level were neutral, $7^{\circ}, 10.12 \pm 0.34$ $\mathrm{mm}$ (range, $10-12 \mathrm{~mm}$ ), and $4.47 \pm 2.17 \mathrm{~mm}$ (range, $2-8$ $\mathrm{mm})$, respectively. The verified actual tibial cutting coronal alignment, sagittal alignment (posterior slope), lateral cutting level, and medial cutting level were $0.04^{\circ} \pm 0.64^{\circ}$ valgus (range, $1^{\circ}$ varus to $1.5^{\circ}$ valgus), $7.19^{\circ} \pm 0.79^{\circ}$ (range, $5.5^{\circ}-8.5^{\circ}$ ), $9.07 \pm 1.45 \mathrm{~mm}$ (range, $5.5-13 \mathrm{~mm}$ ), and 3.48 $\pm 2.00 \mathrm{~mm}$ (range, $1-7.5 \mathrm{~mm}$ ), respectively. The difference between the planned and verified actual cutting levels involving tibial medial and lateral resection was statistically significant $(p<0.05)$ but there was no notable difference 
Jung et al. Appropriateness of the Use of Navigation System in Total Knee Arthroplasty

Clinics in Orthopedic Surgery • Vol. 12, No. 3, $2020 \bullet$ www.ecios.org

\begin{tabular}{|c|c|c|c|c|c|}
\hline \multirow[b]{2}{*}{ Variable } & \multicolumn{3}{|c|}{ Anterior femoral cutting } & \multicolumn{2}{|c|}{ Distal femoral cutting } \\
\hline & Coronal & Sagittal & AP shift & Coronal & $\begin{array}{l}\text { Resection } \\
\text { level }\end{array}$ \\
\hline Planned & Neutral & $1.2^{\circ} \mathrm{E}$ & Neutral & Neutral & $9.08 \mathrm{~mm}$ \\
\hline Verified & $0.09^{\circ} \mathrm{ER}$ & $0.18^{\circ} \mathrm{E}$ & $0.17 \mathrm{~mm}$ Ant & $0.13^{\circ}$ varus & $9.87 \mathrm{~mm}$ \\
\hline $\begin{array}{l}p \text {-value } \\
(<0.05)\end{array}$ & 0.701 & $<0.001$ & 0.098 & 0.074 & $<0.001$ \\
\hline
\end{tabular}

AP: anteroposterior, E: extension, ER: external rotation, Ant: anterior.

Table 2. Planned and Verified Actual Values on Tibial Cutting

\begin{tabular}{|c|c|c|c|c|}
\hline \multirow[b]{2}{*}{ Variable } & \multicolumn{4}{|c|}{ Tibial cutting } \\
\hline & Coronal & Post slope & $\begin{array}{l}\text { Lateral cutting } \\
\text { level }\end{array}$ & $\begin{array}{l}\text { Medial cutting } \\
\text { level }\end{array}$ \\
\hline Planned & Neutral & $7^{\circ}$ & $10.12 \mathrm{~mm}$ & $4.47 \mathrm{~mm}$ \\
\hline Verified & $0.04^{\circ}$ valgus & $7.19^{\circ}$ & $9.07 \mathrm{~mm}$ & $3.48 \mathrm{~mm}$ \\
\hline $\begin{array}{l}p \text {-value } \\
(<0.05)\end{array}$ & 0.65 & 0.092 & $<0.001$ & 0.02 \\
\hline
\end{tabular}

in coronal alignment and posterior slope (Table 2).

At full extension, the average mechanical HKA angle recorded by navigation after the actual implant insertion was $0.23^{\circ} \pm 0.51^{\circ}$ varus (range, $1.5^{\circ}$ varus to $1^{\circ}$ valgus). The medial gap and lateral gap at full extension recorded after insertion of the actual implant were $12.5 \pm 2.1 \mathrm{~mm}$ (range, 8-17.5 mm) and $12.44 \pm 1.85 \mathrm{~mm}$ (range, 8.5-15.5 $\mathrm{mm}$ ), respectively. The average mechanical HKA angle at 3 months postoperatively was $0.45^{\circ} \pm 0.58^{\circ}$ varus (range, $1.8^{\circ}$ varus to $1.2^{\circ}$ valgus). There was no outlier case.

\section{DISCUSSION}

TKA with computed navigation is well known to provide clinical and radiological improvement in the alignment of components. ${ }^{15,16)}$ It was reported to decrease postoperative embolism ${ }^{17)}$ and blood loss even in patients with high BMI. ${ }^{18,19)}$ Lack of evidence suggesting postoperative functional improvement has been one of the factors limiting the extensive use of TKR with computed navigation. Some studies revealed there was no difference in functional outcome between navigation-assisted TKA and traditional TKA. ${ }^{9,20)}$ This could be because outcome assessment measures were gross and not powered adequately to detect statistical difference in most small size studies. These stud- ies were also underpowered for detection of functional improvement. However, there is evidence in the literature (including a meta-analysis) showing that TKR with computed navigation improved anatomical accuracy, which led to functional improvement. ${ }^{15,21,22)}$

In our study, the intraoperatively selected femoral implant size was smaller than the size calculated using the navigation system and measured with the femoral sizing apparatus during the operation. The size of the selected implant was consistent with the size measured using a preoperative template with a plain radiograph. The difference in implant size can be attributed to inadequate removal of osteophytes before registration and the preference of the surgeon for the smaller implant among those readily available.

Accurate acquisition of landmarks and directions is crucial: incorrect acquisition of these pointers or implementation of automatic plans will lead to inaccurate resection levels and sizing. The intraoperative mechanical HKA axis is recorded at the femoral head, knee, and ankle with the navigation system. The starting point of the weightbearing axis (Mikulicz line) has an accuracy of $5 \mathrm{~mm}$, which corresponds to a deviation of approximately $0.7^{\circ}$ with a femur length of $40 \mathrm{~cm}$. The mechanical axis point of the distal end of the femur is recorded at an anterolateral point of the origin of the posterior cruciate ligament. The software uses the femoral mechanical axis point to determine the varus/valgus and the flexion/extension alignment of the femoral resection planes, which in turn affects the overall leg alignment. The pointer tip at the beginning of the anterior cruciate ligament at the anterior intercondylar eminence is also held and pivoted to define the mechanical axis on the tibial plateau. The software uses the points acquired on the medial and lateral malleoli to calculate the midpoint of the talus as the distal endpoint of the tibial mechanical axis. A thicker soft tissue or draping layer around the ankle may alter the result. The foregoing procedures are important to determine the mechanical axis point, and therefore, the landmarks and pivoting steps need to be as accurate as possible. The points defined on the medial and lateral epicondyles in the distal femur define the epicondylar axis, the first of 3 references for the rotational alignment of the femoral implant. This step may determine the initial sizing of the femoral implant. The femoral anterior cortex point provides the reference for the anterior alignment of the implant and also determines the size of the femoral implant. It is important to hold the pointer tip to the anterior cortex near the most proximal point of the implant location and pivot the pointer. The software uses points acquired on the medial and lateral 
Jung et al. Appropriateness of the Use of Navigation System in Total Knee Arthroplasty

Clinics in Orthopedic Surgery • Vol. 12, No. 3, $2020 \bullet$ www.ecios.org

condyles to calculate the most distal and posterior points on the condyles. The most distal and posterior points on the femoral condyles are also used to size the femoral implant and calculate the resection level of the distal resection.

In our study, the average planned sagittal alignment involved $1.2^{\circ}$ extension but was not neutral. It was based on extensive experience with the flexion of the verified angle after incision. The average planned cutting level was 9.08, but the verified actual anterior femoral cutting to obtain balanced flexion and extension gaps was $9.87 \mathrm{~mm}$. For tibial registration, the most medial, lateral, and anterior ends are pointed to completely define the tibial contour. The tibial contour is essential for sizing and positioning of the tibial implant. The software uses the mediolateral distance to determine the corresponding implant size. The AP direction of the tibia determines the direction of the posterior slope and the neutral $\left(0^{\circ}\right)$ rotational alignment of the tibial resection plane. When defining the tibial AP direction, it is important to use the medial one-third of the tibial tubercle. To calculate the tibial resection level, the software uses the medial and lateral tibial plateau points. In our study, there was a significant difference between the planned resection plane and the actual position determined by the navigation instrument. In advanced osteoarthritic knees, bone damage on the medial and lateral plateau may lower the deepest point as the basis of the resection level acquired on high and low tibial plateaus. The tibial resection level should be decided by serial cutting and frequent gap measurement to prevent complications due to unexpected over-resection of the proximal tibia.

This study has several potential limitations. First, although all surgical procedures were performed by a single surgeon, surgical proficiency may have influenced the registration time and execution of the planned resection. After registration, the cutting block adapter was placed on the target. The software recognizes the geometry of the cutting block adapter and calibrates the alignment of the target. The goal was to align the distance between the planned resection plane and the actual position on the navigated instrument to be $0 \mathrm{~mm}$ or $0^{\circ}$. It may have been influenced by the surgeon's skill or the condition of the instrument such as the sawblade, which can be overcome by training or refining the instrument for precision cutting.
In our study, however, the surgeons were experienced with a high volume of operations. Second, we did not consider the effects of sagittal alignment, which might be crucial for implant longevity in TKA. It was possible to precisely quantify sagittal alignment in navigation-assisted surgery. We inserted the real implant when the flexion angle was less than $5^{\circ}$. Third, one of the most important issues of computer navigation is that the index obtained through it may not accurately predict the postoperative condition. At full extension, the average mechanical HKA angle recorded by navigation after insertion of the actual implant was $0.23^{\circ}$ varus, and the average mechanical HKA angle at 3 months postoperatively was $0.45^{\circ}$ varus. In the outlier analysis of the HKA differences, no case was detected to be beyond the acceptable limit. Beneficial radiographic outcomes in TKA might be associated with excellent long-term clinical results. Fourth, cutting amount can be influenced by the sawblade thickness. However, we used the identical set of tools for navigation surgery. The gap or vertical sway between the sawblade and the slot of the cutting apparatus was minimal or absent. Fifth, the cutting procedure can be influenced by the fixation strength of an apparatus. All devices were fixed with multiple screws. Planned values were checked through slots of the apparatus before cutting. The verified values were checked on the cut surface. The changes of the planned values could be also checked through the slots after cutting. But limitations associated with the sawblade thickness and cutting apparatus can also occur in conventional TKA. It should be studied further whether the difference between the planned and verified values of femoral and tibial cutting was due to the registration step on navigation- or execution-related errors. If cutting level differences are due to cutting itself, similar errors can occur in conventional TKA.

In conclusion, it is important for surgeons to be aware of the issues associated with navigation in TKA. The system solely assists surgeon and does not substitute or replace the surgeon's clinical experience or responsibility.

\section{CONFLICT OF INTEREST}

No potential conflict of interest relevant to this article was reported.

\section{REFERENCES}

1. Maloney WJ, Schurman DJ. The effects of implant design on range of motion after total knee arthroplasty. Total con- dylar versus posterior stabilized total condylar designs. Clin Orthop Relat Res. 1992;(278):147-52. 
Jung et al. Appropriateness of the Use of Navigation System in Total Knee Arthroplasty

Clinics in Orthopedic Surgery • Vol. 12, No. 3, $2020 \bullet$ www.ecios.org

2. Yoshioka Y, Siu D, Cooke TD. The anatomy and functional axes of the femur. J Bone Joint Surg Am. 1987;69(6):873-80.

3. Reed SC, Gollish J. The accuracy of femoral intramedullary guides in total knee arthroplasty. J Arthroplasty. 1997;12(6): 677-82.

4. Bathis H, Perlick L, Tingart M, Luring C, Zurakowski D, Grifka J. Alignment in total knee arthroplasty: a comparison of computer-assisted surgery with the conventional technique. J Bone Joint Surg Br. 2004;86(5):682-7.

5. Confalonieri N, Manzotti A, Pullen C, Ragone V. Computer-assisted technique versus intramedullary and extramedullary alignment systems in total knee replacement: a radiological comparison. Acta Orthop Belg. 2005;71(6):703-9.

6. Shi J, Wei Y, Wang S, et al. Computer navigation and total knee arthroplasty. Orthopedics. 2014;37(1):e39-43.

7. Moskal JT, Capps SG, Mann JW, Scanelli JA. Navigated versus conventional total knee arthroplasty. J Knee Surg. 2014; 27(3):235-48.

8. Lee HJ, Lee JS, Jung HJ, Song KS, Yang JJ, Park CW. Comparison of joint line position changes after primary bilateral total knee arthroplasty performed using the navigation-assisted measured gap resection or gap balancing techniques. Knee Surg Sports Traumatol Arthrosc. 2011;19(12):2027-32.

9. Kim YH, Park JW, Kim JS. Computer-navigated versus conventional total knee arthroplasty a prospective randomized trial. J Bone Joint Surg Am. 2012;94(22):2017-24.

10. Cheng T, Zhao S, Peng X, Zhang X. Does computer-assisted surgery improve postoperative leg alignment and implant positioning following total knee arthroplasty? A metaanalysis of randomized controlled trials? Knee Surg Sports Traumatol Arthrosc. 2012;20(7):1307-22.

11. Barrett WP, Mason JB, Moskal JT, Dalury DF, Oliashirazi A, Fisher DA. Comparison of radiographic alignment of imageless computer-assisted surgery vs conventional instrumentation in primary total knee arthroplasty. J Arthroplasty. 2011;26(8):1273-84.

12. Eum DS, Lee HK, Hwang SY, Park JU. Blood loss after navigation-assisted minimally invasive total knee arthroplasty. Orthopedics. 2006;29(10 Suppl):S152-4.

13. Klein GR, Austin MS, Smith EB, Hozack WJ. Total knee arthroplasty using computer-assisted navigation in patients with deformities of the femur and tibia. J Arthroplasty. 2006;
21(2):284-8.

14. van der Linden-van der Zwaag HM, Valstar ER, van der Molen AJ, Nelissen RG. Transepicondylar axis accuracy in computer assisted knee surgery: a comparison of the CTbased measured axis versus the CAS-determined axis. Comput Aided Surg. 2008;13(4):200-6.

15. Hetaimish BM, Khan MM, Simunovic N, Al-Harbi HH, Bhandari M, Zalzal PK. Meta-analysis of navigation vs conventional total knee arthroplasty. J Arthroplasty. 2012;27(6): 1177-82.

16. Lee DH, Park JH, Song DI, Padhy D, Jeong WK, Han SB. Accuracy of soft tissue balancing in TKA: comparison between navigation-assisted gap balancing and conventional measured resection. Knee Surg Sports Traumatol Arthrosc. 2010;18(3):381-7.

17. Kalairajah Y, Cossey AJ, Verrall GM, Ludbrook G, Spriggins AJ. Are systemic emboli reduced in computer-assisted knee surgery? A prospective, randomised, clinical trial. J Bone Joint Surg Br. 2006;88(2):198-202.

18. McConnell J, Dillon J, Kinninmonth A, Sarungi M, Picard F. Blood loss following total knee replacement is reduced when using computer-assisted versus standard methods. Acta Orthop Belg. 2012;78(1):75-9.

19. Millar NL, Deakin AH, Millar LL, Kinnimonth AW, Picard F. Blood loss following total knee replacement in the morbidly obese: effects of computer navigation. Knee. 2011;18(2):10812.

20. Allen CL, Hooper GJ, Oram BJ, Wells JE. Does computerassisted total knee arthroplasty improve the overall component position and patient function? Int Orthop. 2014;38(2): 251-7.

21. Khakha RS, Chowdhry M, Sivaprakasam M, Kheiran A, Chauhan SK. Radiological and functional outcomes in computer assisted total knee arthroplasty between consultants and trainees: a prospective randomized controlled trial. J Arthroplasty. 2015;30(8):1344-7.

22. Alcelik IA, Blomfield MI, Diana G, Gibbon AJ, Carrington N, Burr S. A comparison of short-term outcomes of minimally invasive computer-assisted vs minimally invasive conventional instrumentation for primary total knee arthroplasty: a systematic review and meta-analysis. J Arthroplasty. 2016;31(2):410-8. 